\title{
Contactin-associated protein-like 2 expression in SH-SY5Y cells is upregulated by a FOXP2 mutant with a shortened poly-glutamine tract
}

\author{
YUNJING ZHAO ${ }^{1}$, XIAOLIANG LIU ${ }^{2}$, HONGWEI SUN ${ }^{3}$, YUEPING WANG ${ }^{2}$, \\ WENZHU YANG ${ }^{1}$ and HONGWEI MA ${ }^{1}$
}

\begin{abstract}
Departments of ${ }^{1}$ Developmental Pediatrics and ${ }^{2}$ Clinical Genetics, Shengjing Hospital of China Medical University, Shenyang, Liaoning 110004; ${ }^{3}$ Department of Pediatrics, Central Hospital Affiliated to Shenyang Medical College, Shenyang, Liaoning 110024, P.R. China
\end{abstract}

Received February 11, 2015; Accepted September 14, 2015

DOI: $10.3892 / \mathrm{mmr} .2015 .4483$

\begin{abstract}
The forkhead box protein P2 (FOXP2) gene encodes an important transcription factor that contains a polyglutamine (poly-Q) tract and a forkhead DNA binding domain. It has been observed that FOXP2 is associated with speech sound disorder (SSD), and mutations that decrease the length of the poly-Q tract were identified in the FOXP2 gene of SSD patients. However, the exact role of poly-Q reduction is not well understood. In the present study, constructs expressing wild-type and poly-Q reduction mutants of FOXP2 were generated by polymerase chain reaction (PCR) using lentiviral vectors and transfected into the SH-SY5Y neuronal cell line. Quantitative reverse transcription (qRT)-PCR and western blotting indicated that infected cells stably expressed high levels of FOXP2. Using this cell model, the impact of FOXP2 on the expression of contactin-associated protein-like 2 (CNTNAP2) were investigated, and CNTNAP2 mRNA expression levels were observed to be significantly higher in cells expressing poly-Q-reduced FOXP2. In addition, the expression level of CASPR2, a mammalian homolog of Drosophila Neurexin IV, was increased in cells expressing the FOXP2 mutant. Demonstration of regulation by FOXP2 indicates that CNTNAP2 may also be involved in SSD.
\end{abstract}

\section{Introduction}

Developmental speech and language disorders are highly heritable, with complex multifactorialinheritanceinthe majority of cases (1). A heterozygous mutation of the forkhead-box

Correspondence to: Dr Yunjing Zhao, Department of Developmental Pediatrics, Shengjing Hospital of China Medical University, 36 Sanhao Street, Shenyang, Liaoning 110004, P.R. China E-mail: cmuzyj@163.com

Key words: FOXP2, CNTNAP2, CASPR2, SH-SY5Y cells, polyglutamine, speech sound disorder protein $\mathrm{P} 2$ (FOXP2) gene causes a rare Mendelian speech and language disorder, and was the first speech and language disorder-associated gene to be identified (2). The FOXP2 gene (OMIM, 605317), located on 7q31, encodes a transcription factor that contains a polyglutamine (poly-Q) tract and a forkhead DNA binding domain (2). Previous studies have reported that point mutations and chromosomal abnormalities of FOXP2 are associated with speech and language disorders, which are characterized by difficulties with the learning and production of sequences of oral movements (3-7).

Speech sound disorder (SSD) is the prevalent speech disorder in preschool children, and individuals with SSD have difficulty producing speech sounds appropriate for their age and dialect. In our previous study on a Chinese Han population, it was observed that FOXP2 may confer vulnerability to SSD (8). Deletion of the heterozygous triplet codon, CAA in exon 5 of FOXP2 resulted in five probands presenting with SSD. There are 40 poly-Q tracts encoded by (CAG)4CAA(CAG)4(CAA)2(CAG)2(CAA)2(CAG)3 (CAA)5(CAG)2(CAA)2(CAG)5CAA(CAG)5CAACAG within exon 5. This same heterozygous CAA deletion (c.520delCAA, p.Q174del) was present in five probands, but absent in normal control subjects. The entire mutated sequence (mutation site italicized) is as follows: (CAG)4CAA(CAG)4(CAA)2(CAG)2 (CAA)2(CAG)3(CAA)4(CAG)2(CAA)2(CAG)5CAA(CAG)5 CAACAG. The amino acid, glutamine is encoded by CAG and CAA codons, and the c.520delCAA mutation is predicted to result in a reduction in the length of the poly- $Q$ tract from 40 to 39 residues. Butland et al (9) investigated the length of CAG-poly-Q tracts in the entire human genome in a sample of healthy individuals, and observed that FOXP2 has the longest mean and maximum poly-Q tract length with relatively little variance. Previous studies have demonstrated that the length of CAG-only tracts is correlated with Q-tract variance (10-12) and interruptions in CAG codons may stabilize highly repetitive poly-Q tracts $(10,13)$. Thus, FOXP2 may have increased stability and be less polymorphic with a shorter CAG repeat that is interrupted by CAA codons.

The expansion of poly-Q-encoding CAG codon repeats has been identified as pathogenic in numerous genes associated 
with neurodegenerative disorders, including the HD-associated huntingtin gene in Huntington's disease (14). However, the clinical significance of reductions in poly-Q tracts is unclear. Vernes et al (15) demonstrated thatFOXP2, a transcription factor expressed in neurons, binds to and markedly downregulates the expression of contactin-associated protein-like 2 (CNTNAP2). The CNTNAP2 gene, located on 7q35-36.1, encodes contactin-associated protein 2 (CASPR2), which belongs to the neurexin ion transporter family (16) and is highly expressed in language-associated circuits of the brain (17). CNTNAP2 has been implicated in multiple neurodevelopmental disorders, including autism (18-23), and may affect early language acquisition in normal individuals (24). However, to the best of our knowledge, the potential association between CNTNAP2 and SSD has not been investigated. In the present study, the transcriptional regulation of poly-Q reduction mutations of FOXP2 on CNTNAP2 was investigated.

\section{Materials and methods}

Vector construction. The GV287 lentiviral expression system containing the green fluorescent protein (GFP) coding sequence (Genechem Co., Ltd., Shanghai, China) was used to generate FOXP2 expression vectors. The T-vector-FOXP2 containing wild-type FOXP2 cDNA (NM_014491.3, isoform I) was purchased from OriGene Technologies Inc. (Rockville, MD, USA). The coding sequence of FOXP2 was inserted into the lentiviral vector, GV287 by homologous recombination. The wild-type $F O X P 2$ lentivirus was designated as LV-FOXP2-WT.

The poly-Q reduction (c.520delCAA) FOXP2 mutant vector was generated by four rounds of polymerase chain reaction (PCR) using genomic DNA from an SSD patient that included a heterozygous CAA deletion, as previously described (8). The primer sequences and vector construction strategy are presented in Table I and Fig. 1, respectively. Primers 3 and 6 were used to amplify the poly-Q reduction fragment, and primers 1 and 4 were used to obtain the sequence upstream of $F O X P 2$. A homologous recombination arm was introduced in primer 1 , and primers 2 (also including a homologous recombination arm) and 5 were used to amplify the downstream sequence. The entire FOXP2 coding sequence was subsequently amplified using primers 1 and 2 and the above PCR products ( $\mathrm{a}, \mathrm{b}$ and $\mathrm{c}$ ) as template. The mutant form of FOXP2 was inserted into the lentiviral vector, GV287 by homologous recombination and designated as LV-FOXP2-MT. The empty lentiviral vector, GV287 (termed LV-NC) served as a negative control. All constructed vectors were confirmed by direct sequencing.

Establishment of stably transfected SH-SY5Y cell lines. The SH-SY5Y human cell-line (Genechem Co., Ltd.) was used for expression analysis. Cells were plated at a density of $5 \times 10^{4}$ cells/well on poly-L-lysine-coated 6 -well plates (Sigma-Aldrich, St. Louis, MO, USA) in Dulbecco's modified Eagle's medium/Ham's F12 nutrient mixture (Gibco; Thermo Fisher Scientific, Inc., Waltham, MA, USA) containing 10\% fetal bovine serum (FBS; Gibco; Thermo Fisher Scientific, Inc.) as expansion medium ( $2 \mathrm{ml} /$ well). Cells were grown at $37^{\circ} \mathrm{C}$ in the presence of $5 \% \mathrm{CO}_{2}$. SH-SY5Y cells were infected with the appropriate lentivirus (LV-FOXP2-WT, LV-FOXP2-MT or LV-NC). Three days following infection, the efficiency of lentiviral expression was determined by visualizing the GFP reporter using a MicroPublisher 3.3 RTV microscope (Olympus Corporation, Tokyo, Japan). Stably infected cells were cultured and harvested at $80 \%$ confluence for subsequent RNA and protein extraction. Untreated SH-SY5Y cells served as a blank control (control group).

RNA extraction and quantitative reverse transcription (qRT)-PCR analysis. SH-SY5Y cells from each group were cultured in 12-well plates in expansion medium $(2 \mathrm{ml} /$ well $)$ for three days, and total RNA was extracted using Invitrogen TRIzol reagent (Thermo Fisher Scientific, Inc.). The first strand cDNA was synthesized using Axygen M-MLV-Reverse Transcriptase (Corning Life Sciences, Union City, CA, USA). qRT-PCR was subsequently performed using an Applied Biosystems ABI 7500 system (Thermo Fisher Scientific, Inc.) with the SYBR Green Master mix (Takara Bio, Inc., Otsu, Japan). Primers were synthesized as follows: Forward, 5'-GCGTCAGGGACTCATCTCC-3' and reverse, 5'-GAG GTCTAGCCCTCCATGTTTA-3' for FOXP2; and forward, 5'-TTTAATGGCCCGTTTATGTC-3' and reverse, 5'-TTG TTTCGCACTGGTTTAGG-3' for CNTNAP2; and forward, 5'-TGACTTCAACAGCGACACCCA-3' and reverse, 5'-CAC CCTGTTGCTGTAGCCAAA-3' for GAPDH (Genechem Co., Ltd.). The cycling parameters were as follows: $95^{\circ} \mathrm{C}$ for $10 \mathrm{~min}$, followed by 40 cycles of $95^{\circ} \mathrm{C}$ for $15 \mathrm{sec}, 60^{\circ} \mathrm{C}$ for $1 \mathrm{~min}$, and $72^{\circ} \mathrm{C}$ for $30 \mathrm{sec}$. Dissociation curves were generated to ensure that a single and specific product was amplified. Cycle quantification values $\left(\mathrm{C}_{\mathrm{q}}\right)$ were analyzed by Applied Biosystems SDS1.4 software (Thermo Fisher Scientific, Inc.) and relative quantification of the FOXP2 and CNTNAP2 expression levels was determined using the comparative $\mathrm{C}_{\mathrm{q}}$ method with the GAPDH transcript serving as an internal control.

Western blot analysis. Total protein $(300 \mu \mathrm{g})$ was isolated from infected SH-SY5Y cells and the protein concentration was determined using a BCA Protein assay kit (GE Healthcare Life Sciences, Logan, UT, USA). Equal quantities of protein $(40 \mu \mathrm{g})$ were denatured and separated by $10 \%$ SDS-PAGE (VE-180; Tanon Science \& Technology Co., Ltd., Shanghai, China) at $80 \mathrm{~V}$ for $\sim 2$ h. Gels were transferred to polyvinylidene fluoride membranes (EMD Millipore, Billerica, MA, USA) at $300 \mathrm{~mA}$ for $150 \mathrm{~min}$, membranes were blocked with 5\% milk in Tris-buffered saline Tween-20 (TBST; Genechem Co., Ltd.) buffer and incubated with primary antibodies, rabbit monoclonal anti-FOXP2 (1:1,000; cat. no. 5337; Cell Signaling Technology, Inc., Danvers, MA, USA), rabbit polyclonal anti-CASPR2 (1:1,000; cat. no. 3731; Cell Signaling Technology, Inc.) or mouse monoclonal anti-GAPDH $(1: 10,000$; cat. no. KC-5G4; KangChen Bio-Tech Co. Ltd., Shanghai, China), overnight at $4^{\circ} \mathrm{C}$. Following incubation with goat polyclonal anti-rabbit and anti-mouse immunoglobulin G-horseradish peroxidase secondary antibodies (1:2,000; sc-2004 and sc-2005; Santa Cruz Biotechnology, Inc., Dallas, TX, USA), detection was assessed with an ECL detection kit (Thermo Fisher Scientific, Inc.). Densitometry was conducted using Image 
Table I. Primers used in the present study.

\begin{tabular}{|c|c|c|}
\hline Product & Primer sequence & bp \\
\hline \multirow[t]{2}{*}{ a } & P1 F: 5'-GAGGATCCCCGGGTACCGGTCGCCACCATGATGCAGGAATCTGCGACAG-3' & 378 \\
\hline & P4 R: 5'-CTGCTGAGGAGACAGGACTTG-3' & \\
\hline \multirow[t]{2}{*}{ b } & P3 F: 5'-CAAGTCCTGTCTCCTCAGCAG-3' & 393 \\
\hline & P6 R: 5'-GATGAGTCCCTGACGCTGAAG-3' & \\
\hline \multirow[t]{2}{*}{$\mathrm{c}$} & P5 F: 5'- CTTCAGCGTCAGGGACTCATC-3' & 1457 \\
\hline & P2 R: 5'-TCCTTGTAGTCCATACCTTCCAGATCTTCAGATAAAGGC-3' & \\
\hline \multirow[t]{2}{*}{$\mathrm{d}$} & P1 F: 5'-GAGGATCCCCGGGTACCGGTCGCCACCATGATGCAGGAATCTGCGACAG-3' & 2186 \\
\hline & P2 R:5'-TCCTTGTAGTCCATACCTTCCAGATCTTCAGATAAAGGC-3' & \\
\hline
\end{tabular}

Underlined sections represent the homologous recombination arms. P, primer; F, forward; R, reverse.

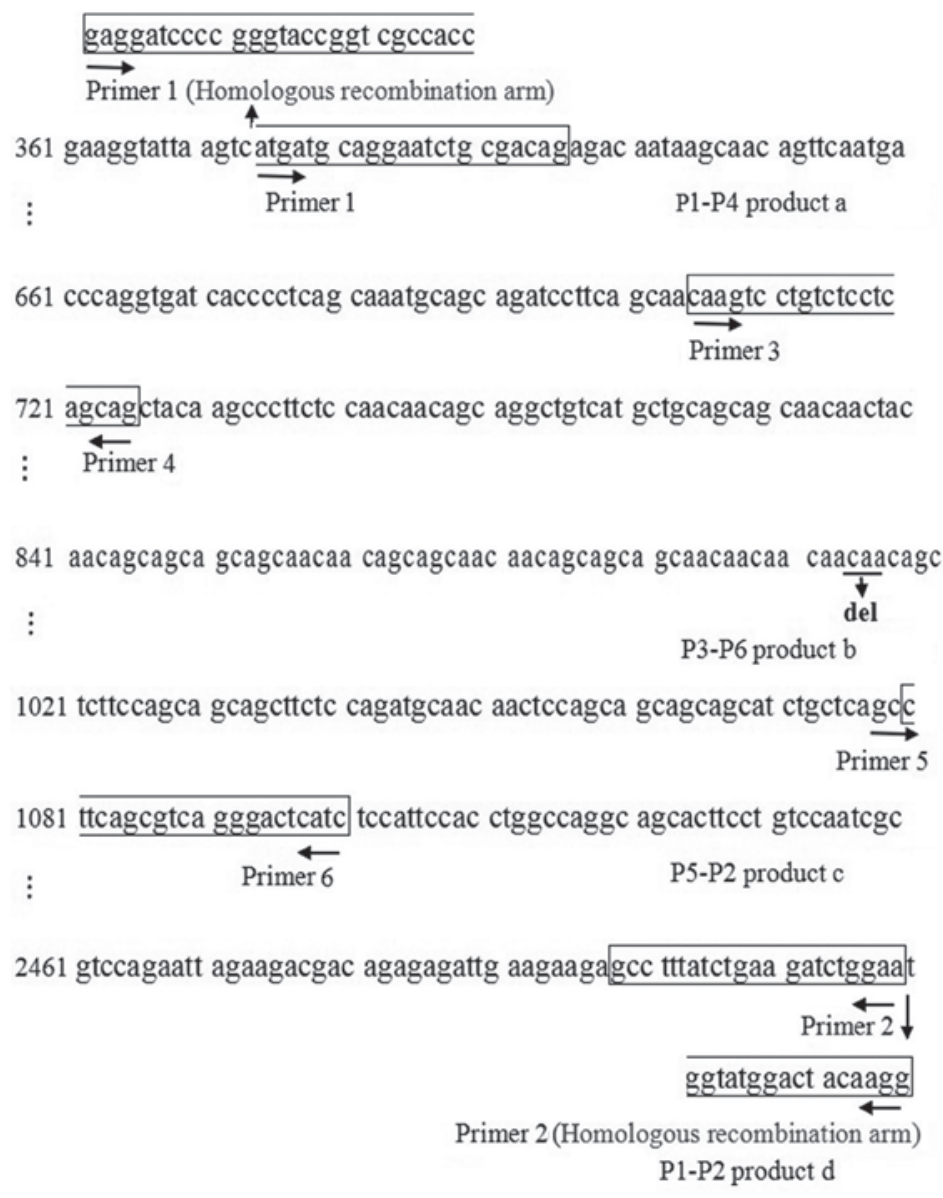

Figure 1. Multiplex polymerase chain reaction synthesis of the FOXP2 poly-glutamine reduction mutant.

J 1.47 software (National Institutes of Health, Bethesda, MD, USA).

Statistical analysis. All analyses were performed using SPSS version 17.0 (SPSS, Inc., Chicago, IL, USA). All data are represented as the mean \pm standard deviation. Post hoc analysis of variance, Student-Newman-Keuls (SNK) test and an independent sample Student's t-test were used to investigate differences in $C N T N A P 2$ expression. $\mathrm{P}<0.05$ was considered to indicate a statistically significant difference.

\section{Results}

Construction of the FOXP2 poly- $Q$ reduction mutant. Exon 5 of FOXP2 is a highly repetitive sequence, which facilitated generation of the poly-Q reduction mutant (c.520delCAA) by multiple rounds of PCR (Fig. 1). The amplified products were visualized by agarose gel electrophoresis and ethidium bromide staining (Fig. 2), and the PCR products were confirmed by sequencing. Reverse sequencing of CAACAG repeats with one CAA deletion confirmed the poly- $\mathrm{Q}$ reduction in the mutant, 


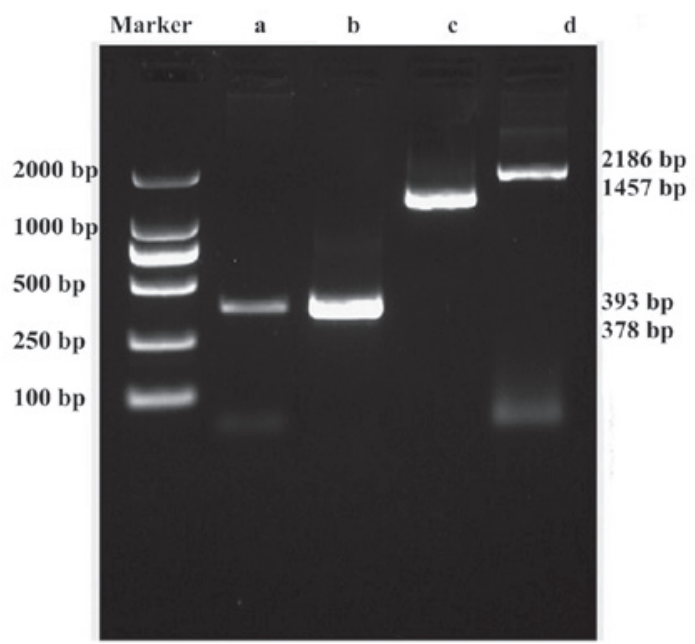

Figure 2. Multiplex polymerase chain reaction products visualized by agarose gel electrophoresis. Gels were stained with ethidium bromide. Lanes a, b, c, and $\mathrm{d}$ correspond to PCR product a, b, c, and d, respectively.

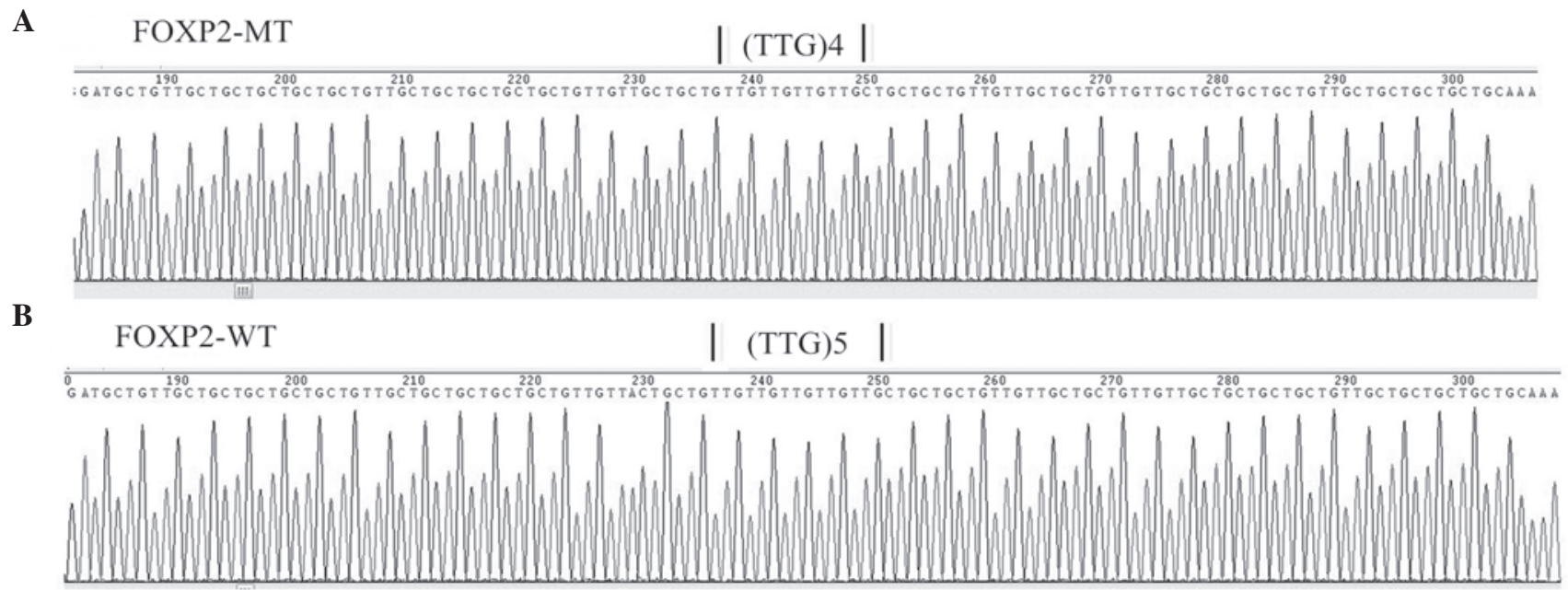

Figure 3. Reverse sequencing of exon 5 identifying (A) the CAA deletion in the mutant FOXP2 gene and (B) the normal wild-type FOXP2 sequence. FOXP2, fork head box protein P2; MT, mutant; WT, wild-type.
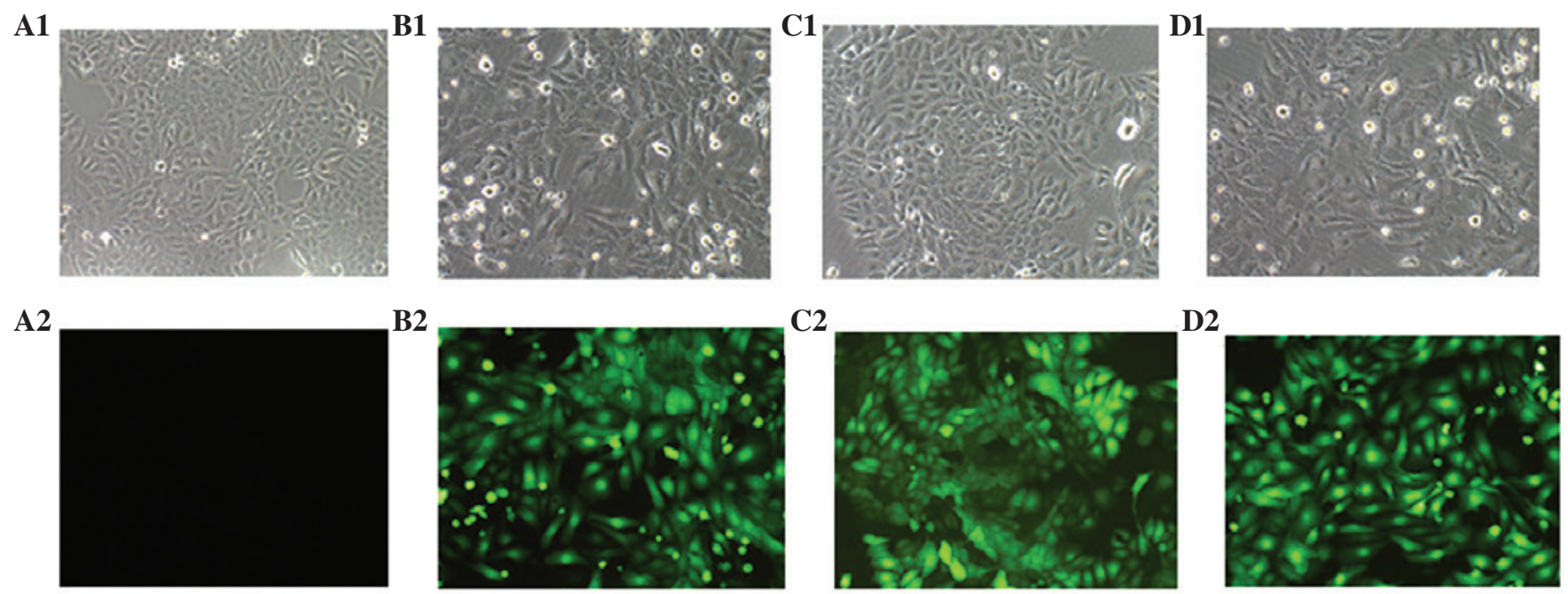

Figure 4. SH-SY5Y cell lines were stably infected with FOXP2-WT and FOXP2-MT and visualized using a visual field microscope (row 1) and fluorescence microscopy (row 2). (A) Untreated SH-SY5Y cells (control group) and cells transfected with (B) LV-NC, (C) LV-FOXP2-WT, and (D) LV-FOXP2-MT. Cells in (B-D) exhibited green fluorescent protein fluorescence, which was not observed in the control group. Magnification, x100. FOXP2, forkhead box protein P2; WT, wild-type; MT, mutant; LV, lentivirus; NC, negative control. 

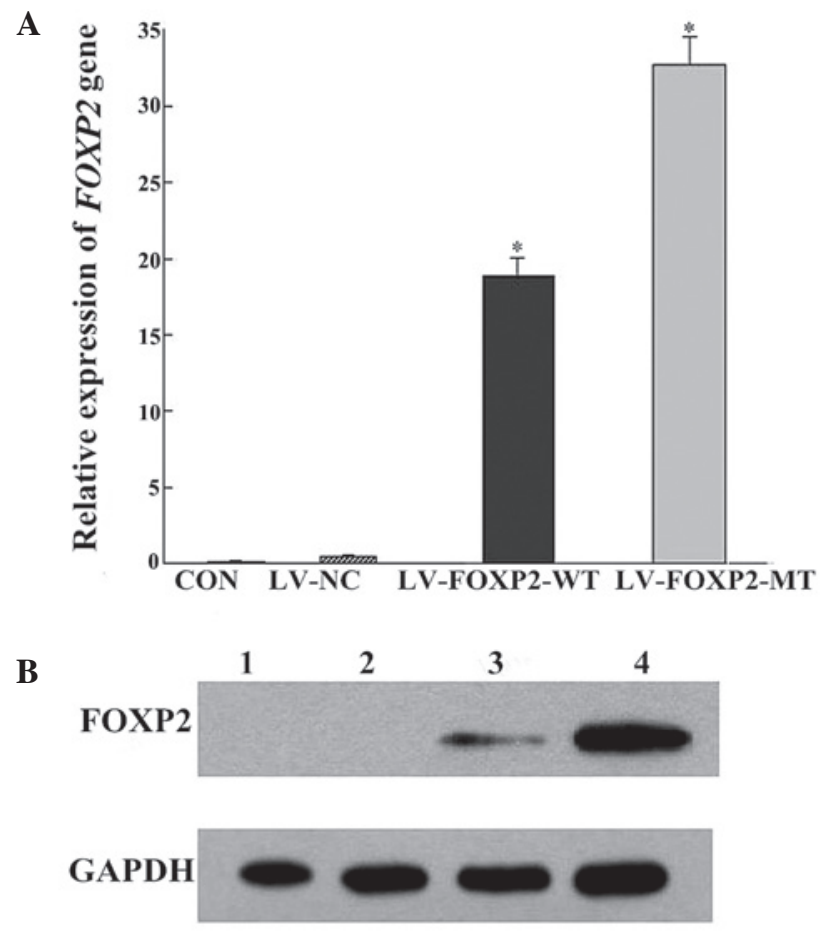

Figure 5. FOXP2 expression in cells transfected with LV-FOXP2-WT and LV-FOXP2-MT as determined by (A) quantitative reverse transcription-polymerase chain reaction $\left({ }^{*} \mathrm{P}<0.05\right.$, vs. the LV-NC) and (B) western blotting ("P $<0.05$, vs. the LV-NC). Lane 1, CON; lane 2, LV-NC; lane 3, LV-FOXP2-WT; and lane 4, LV-FOXP2-MT. FOXP2, forkhead box protein P2; MT, mutant; WT, wild-type; LV, lentivirus; NC, negative control; CON, control.

compared with the wild-type sequence (Fig. 3). The coding sequences of wild-type and mutant $F O X P 2$ were integrated into the GV287 lentiviral expression system by homologous recombination to achieve stable expression of FOXP2.

SH-SY5Y cells were stably infected with LV-FOXP2-WT and $L V$-FOXP2-MT. The human SH-SY5Y cell line is a commonly used cellular model for neuronal function. SH-SY5Y cells were infected with the LV-FOXP2-WT, LV-FOXP2-MT or LV-NC constructs, and expression of GFP was visualized by fluorescence microscopy to determine the efficiency of infection. As presented in Fig. 4, a high intensity of enhanced GFP fluorescence was apparent in the cells infected with all three constructs, however, this was not observed in the untreated cells (control group). A fluorescence ratio of $>80 \%$ was considered to indicate stably transfected cells.

Wild-type FOXP2 and the poly-Q reduction mutant were highly expressed in infected $S H-S Y 5 Y$ cells. FOXP2 expression levels were determined by qRT-PCR, and FOXP 2 mRNA expression levels were significantly higher in SH-SY5Y cells harboring LV-FOXP2-WT and LV-FOXP2-MT constructs $(\mathrm{P}<0.05)$ (Fig. 5A). In addition, FOXP2 protein levels were higher in infected cells, as determined by western blotting (Fig. 5B). These results indicate that the $\mathrm{SH}-\mathrm{SY} 5 \mathrm{Y}$ cell model overexpressed wild-type FOXP2 and the poly-Q reduction mutant.

The FOXP2 poly- $Q$ reduction mutant upregulated the expression of CNTNAP2. In order to investigate the role of
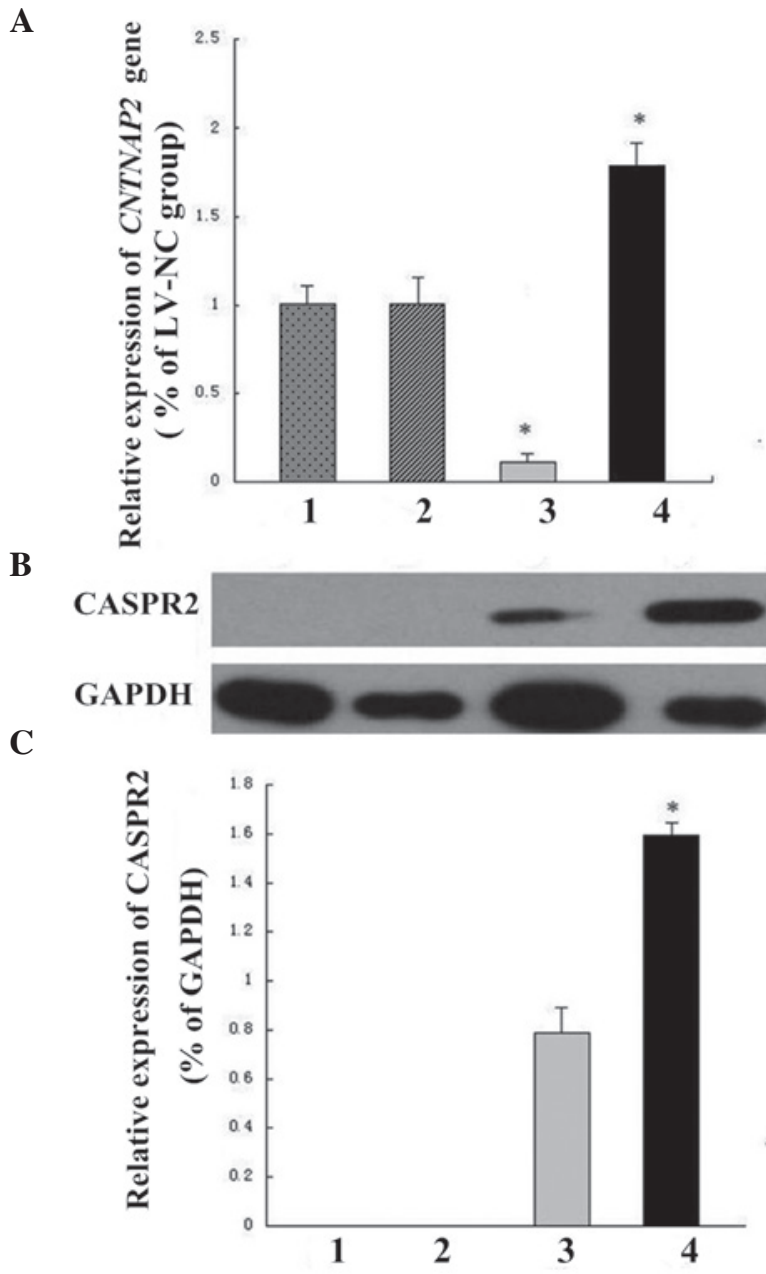

Figure 6. Upregulation of CNTNAP2 expression by FOXP2-MT as determined by qRT-PCR and western blotting. (A) CNTNAP2 transcription identified using qRT-PCR. CNTNAP2 expression level is significantly higher in the cells transfected with LV-FOXP2-MT than in the cells of the CON and LV-NC groups; the CNTNAP2 expression level is significantly lower in cells transfected with LV-FOXP2-WT $\left({ }^{*} \mathrm{P}<0.01\right)$. (B) Analysis of FOXP2 and CASPR 2 protein levels by western blotting. The density of the band corresponding to CASPR2 in the SH-SY5Y-FOXP2-MT cells is markedly higher than that in the SH-SY5Y-FOXP2-WT cells. Lane 1, CON; lane 2, LV-NV; lane 3, LV-FOXP2-WT; and lane4, LV-FOXP2-MT. (C) The level of CASPR2 expression in the LV-FOXP2-MT group is significantly higher than that in the LV-FOXP2-WT group ( $\mathrm{P}<0.01)$. CNTNAP2, contactin-associated protein-like 2; LV, lentivirus; NC, negative control; CASPR2, contactin-associated protein-like 2; FOXP2, forkhead box protein P2; MT, mutant; qRT-PCR, quantitative reverse transcription-polymerase chain reaction; CON, control; WT, wild-type.

poly-Q reduction on CNTNAP2 expression levels, CNTNAP2 mRNA expression levels in the stably infected cell lines were determined by qRT-PCR. CNTNAP 2 mRNA levels were observed to be 0.105 -fold lower in cells infected with LV-FOXP2-WT than those infected with LV-NC, indicating inhibition of CNTNAP2 expression by wild-type FOXP2 (Fig. 6A). In addition, CNTNAP2 mRNA expression levels were 1.788-fold higher in cells infected with LV-FOXP2-MT than those infected with LV-NC, and 17.03-fold higher than those infected with LV-FOXP2-WT. These results indicate that poly-Q reduction of FOXP2 reversed the suppressive effect of the wild-type protein and upregulated transcription of CNTNAP2. 
The levels of the CASPR2 protein, encoded by CNTNAP2, were subsequently determined by western blotting, and CASPR2 expression was not observed in the untreated SH-SY5Y cells (control) or cells infected with LV-NC (Fig. 6B). However, low expression levels of CASPR2 were detected in cells infected with LV-FOXP2-WT, and significantly higher expression levels of CASPR 2 were demonstrated in cells infected with LV-FOXP2-MT $(\mathrm{P}<0.01)$, indicating an upregulation of CNTNAP2 by the FOXP2 poly-Q reduction mutant (Fig. 6C).

\section{Discussion}

In the present study, the SH-SY5Y human neuroblastoma cell line was engineered to stably express wild-type FOXP2 and a poly-Q reduction mutant. Using these cell lines, the effects of poly-Q reduction on the FOXP2-mediated regulation of CNTNAP2 was assessed, and the FOXP2 mutant containing a shorter poly-Q tract was demonstrated to upregulate the expression of CNTNAP2 at the mRNA and protein levels.

The FOXP2 gene encodes an important nervous system transcription factor that includes a long poly-Q tract in exon 5 that varies in length between 34 and 40 glutamine residues (9). However, the physiological consequences of mutations in this poly-Q tract are not well understood. Wassink et al (25) previously detected small internal deletions in the Q40 tract in two families with autism. MacDermot et al (3) reported a heterozygous insertion of the sequence, CAGCAGCAACAA into the poly-Q region of exon 5 in a proband with developmental verbal dyspraxia. In our previous study the poly-Q coding region of FOXP2 in SSD patients from a Chinese Han population was sequenced, and it was observed that a single CAA codon was deleted, which decreased the number of glutamines from 40 to 39 (8). The underlying mechanism by which FOXP2 poly-Q reduction affects SSD requires further investigation.

Identification of putative target genes that may interact with or be regulated by the FOXP2 neural transcription factor is important. The CNTNAP2 gene encodes the CASPR 2 protein, which is highly abundant in the frontal and temporal lobes, in the striatal circuits of the developing human brain, and in the frontal cortex of the adult brain (17). These regions are involved in speech and language learning. Vernes et al (15) reported that FOXP2 markedly downregulates the expression of CNTNAP2. The effects of FOXP2 poly-Q reduction on the expression of CNTNAP2 in SH-SY5Y cells, which have particularly low endogenous FOXP2 expression levels (26), was also investigated in the current study. The cells were stably infected with wild-type and mutant FOXP2 expression vectors using a lentiviral expression system, and GFP fluorescence was used to confirm a successful infection and compare the expression levels. Expression of wild-type and mutant FOXP2 was indicated at the mRNA and protein levels. However, the expression of mutant FOXP2 was significantly higher than that of the wild-type protein, for reasons that remain to be elucidated, however, post-transcriptional regulation, such as mRNA degradation may contribute to this variance. The expression level of CNTNAP2 was significantly decreased in cells expressing wild-type FOXP2 compared with the non-expressing controls, which was consistent with a previous study (15). Notably, the expression level of CNTNAP2 was significantly higher in cells expressing the FOXP2 poly-Q reduction mutant, which reversed the repressive effect of the wild-type protein, and thus markedly upregulated the level of CNTNAP2 expression. To the best of our knowledge, the present study is the first to demonstrate that this divergence in the regulatory activity of wild-type and mutant FOXP2 on CNTNAP2 expression, may underlie the pathogenic mechanism by which poly-Q reduction is associated with SSD. Furthermore, these results indicate a possible association between CNTNAP2 and SSD.

The method by which deletion of a single glutamine from the poly-Q tract of FOXP2 markedly affects the transcription and expression of CNTNAP2 remains to be elucidated. According to a previous study (27), the number of repeats in the FOXP2 poly-Q tract is relatively monomorphic in a healthy population, and in patients presenting with severe speech and language disorders, although 40 is considered to be a large number of repeats, therefore, greater variability may be expected. Sequences may include up to $14 \mathrm{CAA}$ interruptions among the CAG repeats, which prevent slippage and expansion. Therefore, deletion of a CAA codon may decrease the stability of poly-Q tracts, which has been observed to form a hairpin structure in FOXP2 transcripts (27). Deletion of CAA may alter the configuration of the hairpin, which may affect the binding of FOXP2 to the CNTNAP2 promoter region.

In the present study, the results of the qRT-PCR experiments demonstrate CNTNAP2 was expressed in the CON and LV-NC cells, however, the CASPR2 protein was not observed in these cells in the western blotting experiments. By contrast, the CASPR2 protein was detected in SH-SY5Y cells transfected with wild-type FOXP2 and the poly-Q mutant. The reason for this discrepancy has not yet been elucidated, and it has not, to the best of our knowledge, been reported whether CASPR2 is expressed in SH-SY5Y cells. CNTNAP2 is a target of FOXP2, by which it is directly regulated (15). CASPR2 expression may be switched off in the absence of FOXP2, however when present, FOXP2 initiates transcription of CNTNAP2 and subsequent accumulation of CASPR2.

In conclusion, the present study demonstrates that CNTNAP2 expression was significantly upregulated in SH-SY5Y cells expressing a poly-Q reduction mutation of FOXP2. CNTNAP2 may, therefore, also be associated with SSD. Further in vivo investigations using animal models are required to clarify the exact role of poly-Q reduction of FOXP2 in SSD.

\section{Acknowledgements}

The present study was supported by a grant from the National Natural Science Foundation of China (grant nos. 81101019 and 81100187).

\section{References}

1. Fisher SE, Lai CS and Monaco AP: Deciphering the genetic basis of speech and language disorders. Annu Rev Neurosci 26:57-80, 2003.

2. Lai CS, Fisher SE, Hurst JA, Vargha-Khadem F and Monaco AP: A forkhead-domain gene is mutated in a severe speech and language disorder. Nature 413: 519-523, 2001. 
3. MacDermot KD, Bonora E, Sykes N, Coupe AM, Lai CS Vernes SC, Vargha-Khadem F, McKenzie F, Smith RL, Monaco AP and Fisher SE: Identification of FOXP2 truncation as a novel cause of developmental speech and language deficits. Am J Hum Genet 76:1074-1080, 2005.

4. Shriberg LD, Ballard KJ, Tomblin JB, Duffy JR, Odell KH and Williams CA: Speech, prosody, and voice characteristics of a mother and daughter with a 7;13 translocation affecting FOXP2. J Speech Lang Hear Res 49: 500-525, 2006.

5. Feuk L, Kalervo A, Lipsanen-Nyman M, Skaug J, Nakabayashi K, Finucane B, Hartung D, Innes M, Kerem B, Nowaczyk MJ, et al: Absence of a paternally inherited FOXP2 gene in developmental verbal dyspraxia. Am J Hum Genet 79: 965-972, 2006.

6. Zeesman S, Nowaczyk MJ, Teshima I, Roberts W, Cardy JO, Brian J, Senman L, Feuk L, Osborne LR and Scherer SW: Speech and language impairment and oromotor dyspraxia due to deletion of 7q31 that involves FOXP2. Am J Med Genet A 140: 509-514, 2006.

7. Lennon PA, Cooper ML, Peiffer DA, Gunderson KL, Patel A, Peters S, Cheung SW and Bacino CA: Deletion of 7q31.1 supports involvement of FOXP2 in language impairment:clinical report and review. Am J Med Genet A 143 A:791-798, 2007.

8. Zhao Y, Ma H, Wang Y, Gao H, Xi C, Hua T, Zhao Y and Qiu G: Association between FOXP2 gene and speech sound disorder in Chinese population. Psychiatry Clin Neurosci 64: 565-573, 2010

9. Butland SL, Devon RS, Huang Y, Mead CL, Meynert AM, Neal SJ, Lee SS, Wilkinson A, Yang GS, Yuen MM, et al: CAG-encoded polyglutamine length polymorphism in the human genome. BMC Genomics 8: 126, 2007.

10. Jodice C, Giovannone B, Calabresi V, Bellocchi M, Terrenato L and Novelletto A: Population variation analysis at nine loci containing expressed trinucleotide repeats. Ann Hum Genet 61: 425-438,1997.

11. Wren JD, Forgacs E, Fondon JW III, Pertsemlidis A, Cheng SY Gallardo T, Williams RS, Shovet RV, Minna JD and Garner HR: Repeat polymorphisms within gene regions: Phenotypic and evolutionary implications. Am J Hum Genet 67: 345-356, 2000.

12. Mularoni L, Guigó R and Albà MM: Mutation patterns of amino acid tandem repeats in the human proteome. Genome Biol 7: R33, 2006.

13. Sobczak K and Krzyzosiak WJ: Patterns of CAG repeat interruptions in SCA1 and SCA2 genes in relation to repeat instability. Hum Mutat 24: 236-247, 2004.

14. MacDonald M: the Huntington's Disease Collaborative Research Group: A novel gene containing a trinucleotide repeat that is expanded and unstable on Huntington's disease chromosomes. Cell 72: 971-983, 1993.

15. Vernes SC, Newbury DF, Abrahams BS, Winchester L, Nicod J, Groszer M, Alarcón M, Oliver PL, Davies KE, Geschwind DH, et al A functional genetic link between distinct developmental language disorders. N Engl J Med 359: 2337-2345, 2008.
16. Nakabayashi K and Scherer SW: The human contactin-associated protein-like 2 gene (CNTNAP2) spans over $2 \mathrm{Mb}$ of DNA at chromosome 7q35. Genomics 73: 108-112, 2001.

17. Abrahams BS, Tentler D, Perederiy JV, Oldham MC, Coppola G and Geschwind DH: Genome-wide analyses of human perisylvian cerebral cortical patterning. Proc Natl Acad Sci USA 104: 17849-17854, 2007.

18. Alarcón M, Abrahams BS, Stone JL, Duvall JA, Perederiy JV, Bomar JM, Sebat J, Wigler M, Martin CL, Ledbetter DH, et al: Linkage, association, and gene-expression analyses identify CNTNAP2 as an autism-susceptibility gene. Am J Hum Genet 82: 150-159, 2008.

19. Arking DE, Cutler DJ, Brune CW, Teslovich TM, West K, Ikeda M, Rea A, Guy M, Lin S, Cook EH Jr, et al: A common genetic variant in the neurexin superfamily member CNTNAP2 increases familial risk of autism. Am J Hum Genet 82: 160-164, 2008.

20. Rossi E, Verri AP, Patricelli MG, Destefani V, Ricca I, Vetro A, Ciccone R, Giorda R, Toniolo D, Maraschio P and Zuffardi O: A $12 \mathrm{Mb}$ deletion at 7q33-q35 associated with autism spectrum disorders and primary amenorrhea. Eur J Med Genet 51: 631-638, 2008.

21. Bakkaloglu B, O'Roak BJ, Louvi A, Gupta AR, Abelson JF, Morgan TM, Chawarska K, Klin A, Ercan-Sencicek AG, Stillman AA, et al: Molecular cytogenetic analysis and resequencing of contactin associated protein-like 2 in autism spectrum disorders. Am J Hum Genet 82:165-173, 2008.

22. Jackman C, Horn ND, Molleston JP and Sokol DK: Gene associated with seizures, autism and hepatomegaly in an Amish girl. Pediatr Neurol 40: 310-313, 2009.

23. Li X, Hu Z, He Y, Xiong Z, Long Z, Peng Y, Bu F, Ling J, Xun G, Mo X, et al: Association analysis of CNTNAP2 polymorphisms with autism in the Chinese Han population. Psychiatr Genet 20: $113-7,2010$

24. Whitehouse AJ, Bishop DV, Ang QW, Pennell CE and Fisher SE: CNTNAP2 variants affect early language development in the general population. Genes Brain Behav 10: 451-456, 2011.

25. Wassink TH, Piven J, Vieland VJ, Pietila J, Goedken RJ, Folstein SE and Sheffield VC: Evaluation of FOXP2 as an autism susceptibility gene. Am J Med Genet 114: 566-569, 2002.

26. Konopka G, Bomar JM, Winden K, Coppola G, Jonsson ZO, Gao F, Peng S, Preuss TM, Wohlschlegel JA and Geschwind DH: Human-specific transcriptional regulation of CNS development genes by FOXP2. Nature 462: 213-217, 2009.

27. Sobczak K and Krzyzosiak WJ: CAG repeats containing CAA interruptions form branched hairpin structures in spinocerebellar ataxia type 2 transcripts. J Biol Chem 280: 3898-3910, 2005. 